\title{
Vascular endothelial growth factor controls neuronal migration and cooperates with Sema3A to pattern distinct compartments of the facial nerve
}

\author{
Quenten Schwarz, ${ }^{1}$ Chenghua Gu, ${ }^{2,3}$ Hajime Fujisawa, ${ }^{4}$ Kimberly Sabelko, ${ }^{5}$ Marina Gertsenstein, ${ }^{6}$ \\ Andras Nagy, ${ }^{6}$ Masahiko Taniguchi, ${ }^{7}$ Alex L. Kolodkin, ${ }^{2}$ David D. Ginty, ${ }^{2,3}$ David T. Shima, ${ }^{8}$ and \\ Christiana Ruhrberg ${ }^{1,9}$ \\ ${ }^{1}$ Department of Cell Biology, Institute of Ophthalmology, University College London, London EC1V 9EL, United Kingdom; \\ ${ }^{2}$ Department of Neuroscience and ${ }^{3}$ Howard Hughes Medical Institute, The Johns Hopkins University School of Medicine, \\ Baltimore, Maryland 21205, USA; ${ }^{4}$ Group of Developmental Neurobiology, Division of Biological Science, Nagoya \\ University Graduate School of Science, Chikusa-ku, Nagoya 464-8602, Japan; ${ }^{5}$ Department of Neuroscience, University of \\ Pennsylvania School of Medicine, Philadelphia, Pennsylvania 19104, USA; ${ }^{6}$ Samuel Lunenfeld Research Institute, Mount \\ Sinai Hospital, Toronto, Ontario M5G 1X5, Canada; ${ }^{7}$ Department of Biochemistry and Molecular Biology, Graduate School \\ of Medicine, The University of Tokyo, Bunkyo-ku, Tokyo 113-0033, Japan; ${ }^{8}$ Eyetech Pharmaceuticals Inc., \\ Woburn, Massachusetts 01801, USA
}

Developing neurons accurately position their somata within the neural tube to make contact with appropriate neighbors and project axons to their preferred targets. Taking advantage of a collection of genetically engineered mouse mutants, we now demonstrate that the behavior of somata and axons of the facial nerve is regulated independently by two secreted ligands for the transmembrane receptor neuropilin 1 (Nrp1), the semaphorin Sema3A and the VEGF164 isoform of Vascular Endothelial Growth Factor. Although Sema3A is known to control the guidance of facial nerve axons, we now show that it is not required for the pathfinding of their somata. Vice versa, we find that VEGF164 is not required for axon guidance of facial motor neurons, but is essential for the correct migration of their somata. These observations demonstrate, for the first time, that VEGF contributes to neuronal patterning in vivo, and that different compartments of one cell can be co-ordinately patterned by structurally distinct ligands for a shared receptor.

[Keywords: Neuronal migration; axon guidance; neuropilin; VEGF; semaphorins]

Supplemental material is available at http://www.genesdev.org.

Received May 16, 2004; revised version accepted September 13, 2004.

Differentiating neurons position themselves in defined locations along the anterior-posterior and dorso-ventral axes of the developing central nervous system and project axons and dendrites in stereotypical patterns to receive appropriate inputs and send signals to their preferred targets. Mutations that either impair the positioning of cell bodies in the neural tube or disrupt axon guidance in the periphery have disastrous consequences for the function and survival of motor neurons (e.g., Studer et al. 1996; Tiret et al. 1998; Gavalas et al. 2003).

The spatial guidance of axons and dendrites is regulated by an interplay of attractive and repulsive cues, which are present in the extracellular environment of developing neurons and are sensed by signal transducing receptors on filopodia-studded growth cones (for review,

${ }^{9}$ Corresponding author.

E-MAIL c.ruhrberg@ucl.ac.uk; FAX 44-0-207-608-6810.

Article and publication are at http://www.genesdev.org/cgi/doi/10.1101/ gad.322904. see Tessier-Lavigne and Goodman 1996; Dickson 2002). Among the numerous proteins that control axon pathfinding, the transmembrane receptors neuropilin 1 (Nrp1) and neuropilin 2 (Nrp2) play prominent roles because of their high affinity for guidance cues of the semaphorin family (for review, see Raper 2000). The beststudied member of the semaphorin family is Sema3A, a secreted protein that modulates cytoskeletal dynamics, endocytosis, and axonal transport in neurons by activating receptor complexes that contain Nrp1 as the ligandbinding subunit and a member of the plexin family as the signaling subunit (for review, see Castellani and Rougon 2002). Accordingly, loss of Nrp1 abrogates the growth cone collapse of cultured sensory neurons in response to Sema3A, and like loss of Sema3A, causes defasciculation of several cranial and limb nerves (Kitsukawa et al. 1997; Taniguchi et al. 1997).

The spatial cues and signaling pathways that direct the positioning of neuronal cell bodies (somata) in the brain 
are less well defined; however, much progress has recently been made through the analysis of hereditary neuropathologies in humans and mice (for review, see Rice and Curran 2001). The perhaps best-studied genetic pathway controlling neuronal migration is centered around reelin, a secreted protein that binds to the very-low-density lipoprotein receptor (Vldlr) and the apolipoprotein E receptor 2 (ApoER2) to activate the intracellular adaptor disabled (Dab1). The reelin pathway cooperates with a signaling cascade involving Cdk5, a major neuronal kinase implicated in the control of cytoskeletal dynamics. Cdk5 can phosphorylate neurofilaments and several proteins affected in human neurological disorders, most notably doublecortin and the Lissencephaly gene LIS1 (for reviews, see Feng and Walsh 2001; Ohshima and Mikoshiba 2002). LIS1, in turn, can interact with dynein motors, possibly to regulate the transport of the nucleus and other cytoplasmic contents during neuronal migration. Consistent with a role for dynein motors in neuronal migration, we have recently shown that point mutations in cytoplasmic dynein perturb neuronal migration of facial branchiomotor neurons (Hafezparast et al. 2003). Other pathways implicated in the control of neuronal migration contain the secreted netrin or slit proteins and their receptors. For example, loss of function for netrin impairs the migration of neurons of the inferior olive and the pontine nuclei (Bloch-Gallego et al. 1999; Yee et al. 1999).

In addition to their roles in axon guidance, $\mathrm{Nrp} 1$ and Nrp2 have been implicated in the control of neuronal migration. Both proteins are required for the correct pathfinding of GABA-containing interneurons in the telencephalon, and the expression patterns of their semaphorin ligands Sema3A and Sema3F are consistent with a role in guiding this migration (Marin et al. 2001; Tamamaki et al. 2003). However, it is not known if Nrp1 contributes to both soma and axon guidance of the same neuron. To address this question, we examined the development of facial branchiomotor neurons, as they express Nrp1 to control their axon behavior (Kawakami et al. 1996; Kitsukawa et al. 1997) and undergo an extensive migration from their birthplace to the sites of facial motor nucleus assembly (e.g., see Altman and Bayer 1982; Auclair et al. 1996; Gavalas et al. 2003).

Facial branchiomotor neurons are born in a ventral position in a hindbrain segment termed rhombomere $(\mathrm{r})$ 4. Concomitant with the extension of axons into the second branchial arch, they move their somata caudally to travel through $\mathrm{r} 5$ and into $\mathrm{r} 6$, where they move to the opposite side of the basal plate. This migration spans several days, covers many cell diameters, and culminates in the formation of the paired motor nuclei of the VIIth cranial (facial) nerve, which controls movement of the facial musculature. The soma migration of facial branchiomotor neurons can be divided into two distinct phases, a tangential and a radial migration (Fig. 1A). The movement from the ventricular side to the pial side of the hindbrain follows the more common pattern of radial migration, where neurons migrate from the ventricular zone toward the outer surface of the brain in close association with radial glia; in contrast, the earlier movement from $\mathrm{r} 4$ through $\mathrm{r} 5$ and into $\mathrm{r} 6$ is a tangential migration, as it occurs perpendicular to the radial glia scaffold (for review, see Marin and Rubenstein 2003).

We found that loss of Nrp1 compromised the soma migration of facial branchiomotor neurons during the tangential phase and resulted in the formation of misshapen and malpositioned facial motor nuclei. Two semaphorins with a high affinity for Nrp1, Sema3A and Sema3C, were expressed in the hindbrain in a spatiotemporal pattern consistent with a role in the migration of facial branchiomotor somata. However, to our surprise, neither Nrp1 ligand was essential for this process, nor was the ability of Nrp1 to bind semaphorins. Rather, soma migration of facial branchiomotor neurons relied on the presence of a structurally unrelated Nrp1 ligand that is better known for its effect on the growth, differentiation, and patterning of blood vessels, an isoform of Vascular Endothelial Growth Factor (VEGF) termed VEGF164 (Soker et al. 1998; for review, see Ruhrberg 2003).

Our observations suggest that the dual affinity of Nrp1 for VEGF and semaphorin ligands allows facial branchiomotor neurons to coordinate the migratory behavior of their axons and somata: Whereas Sema3A is required for their axon guidance (Taniguchi et al. 1997), VEGF164 supports the correct positioning of their somata within the neural tube. These findings establish, for the first time, that VEGF is required to control the complex behavior of differentiated neurons in vivo, and they have widespread implications for our understanding of signaling pathways implicated in human neurological disorders.

\section{Results}

Nrp1 controls the migration of facial branchiomotor neurons

Using a previously characterized probe for in situ hybridization (He and Tessier-Lavigne 1997), we confirmed that Nrp1 was expressed by facial branchiomotor neurons; moreover, we found that it was expressed by these neurons both during the time of soma migration and after the somata had condensed into nuclei (Supplementary Fig. S1). To examine if Nrp1 signaling was required for the pathfinding of facial branchiomotor somata, we followed their journey in Nrp1-deficient mouse embryos with a marker for postmitotic motor neurons, an in situ probe for the LIM homeodomain protein Isl1 (Ericson et al. 1992).

We found that loss of Nrp1 did not impair the generation of facial branchiomotor neurons in $\mathrm{r} 4$, but that it disrupted the tangential migration of their somata in 13/ 13 cases examined (Fig. 1B-K). More specifically, loss of Nrp1 delayed soma migration and resulted in the separation of the migratory stream into several distinct streams on the ventricular side of the hindbrain (Fig. $1 C, G$, arrowheads). Consequently, many somata initi- 
ated their radial migration toward the pial side of the hindbrain in ectopic anterior locations (Fig. 1E,I) and contributed to the formation of elongated or dumbbellshaped nuclei, rather than the characteristic rounded nuclei seen in wild-type littermates (Fig. 1, cf. H and I, J and $\mathrm{K}$; data not shown). In severe cases, the entire nucleus was shifted anteriorly (Fig. 1, cf. the relative position of facial and trigeminal nuclei in $\mathrm{D}$ and $\mathrm{E}$ ).

The abnormal soma migration of facial branchiomotor neurons is not explained by vascular defects in the hindbrain or axon defects in the periphery

Loss of Nrp1 alters the migratory behavior of facial nerve axons, as they defasciculate once they reach their target area, the second branchial arch (Kitsukawa et al. 1997). Because facial branchiomotor neurons translocate their somata at a time when their axons navigate in the periphery, we asked if the abnormal soma behavior was secondary to the disturbance of axonal patterning. To answer this question, we cultured 11.5-dpc (days postcoitum) hindbrains that had been axotomized and were free of branchial arch tissue under serum-free conditions. We found that removing distal axons or peripheral targets did not compromise soma migration in any of 15

Figure 1. Nrp1 is required for the correct pathfinding of facial branchiomotor somata. (A) Schematic representation of the spatial relationship of trigeminal, facial, abducens, and glossopharyngeal motor neurons in a flat-mounted mouse hindbrain. The axon exit points for trigeminal and facial branchiomotor neurons (hatched circles) and the floorplate (gray) are indicated. The cross-sections on the right-hand side show the position of facial branchiomotor somata on the ventricular (v) side in $\mathrm{r} 4$ (top section) and on the pial (p) side in r6 (bottom section). (B,C) The migration of Isl1-positive facial branchiomotor somata (VIIm) from $\mathrm{r} 4$ to $\mathrm{r} 6$ was observed on the ventricular side in the presence $(B)$ or absence $(C)$ of Nrp1 at $12.5 \mathrm{dpc}$. Wild-type somata stayed loosely associated to form one continuous stream, but mutant somata separated into several distinct streams (arrows and arrowheads indicate separation in $\mathrm{r} 4$ and $\mathrm{r} 5 / \mathrm{r} 6$, respectively). ( $D)$ In the presence of Nrp1, many facial somata have reached the pial side of the hindbrain by $12.5 \mathrm{dpc}$ and formed facial motor nuclei (VIIn). (E) In the absence of Nrp1, many mutant somata emerged on the pial side in an ectopic anterior location (star) instead of their normal location $(\Delta)$. Panel $D$ shows one side of a wild-type hindbrain, and panel $E$ shows the opposite side of a stage-matched mutant hindbrain to highlight differences in the position of facial branchiomotor neurons relative to the trigeminal nucleus $(\mathrm{Vn})$. ( $F)$ Few facial branchiomotor somata were normally present on the ventricular side at 13.5 dpc (bracket). (G) In contrast, many mutant somata were still migrating on the ventricular side at $13.5 \mathrm{dpc}$ (arrowheads). (I) On the pial side, some mutant somata had contributed to normally positioned nuclei (VIIn), while others had formed an ectopic nucleus (star). One side of a wild-type hindbrain $(F, G)$ and the opposite side of a stage-matched mutant hindbrain $(H, I)$ are shown. $(J, K)$ By $14.5 \mathrm{dpc}$, all facial branchiomotor somata have integrated into nuclei on the pial side, which in wild types appeared round $(J)$, but in mutant littermates were usually dumbbell-shaped, with an ectopic anterior component (star; $K$ ). Bars, $250 \mu \mathrm{m}$ (one for $B, C$; one for $D, E$; one for $F-I$; and one for $J, K)$. The midline is indicated with an asterisk. wild-type hindbrain explants (Fig. 2A-D). In contrast, after $2 \mathrm{~d}$ in culture, 14/14 explants lacking Nrp1 contained facial branchiomotor somata in an anterior position (cf. Fig. 2I-L). Taken together, the observations from explant culture experiments suggest that soma migration in the neural tube and axon guidance in the periphery are regulated independently.

The radial glia scaffold assists the radial migration of neurons in the central nervous system, but it is not known if specific structural features support the tangential migration of neurons. Because mutants lacking Nrp1 are unable to form a subventricular vascular plexus (Kawasaki et al. 1999; Q. Schwarz and C. Ruhrberg, unpubl.), and the tangential migration of facial branchiomotor somata occurs in close proximity to the subventricular vascular network (see Fig. 2E; Q. Schwarz and C. Ruhrberg, unpubl.), we asked if their abnormal migration was caused indirectly by the vascular defect. This was not the case, as the soma migration of facial branchiomotor neurons proceeded normally in hindbrain explant cultures, in which vessel networks rapidly degenerated (Fig. 2E-H). Moreover, the somata of facial branchiomotor neurons migrated normally in $2 / 2$ hindbrains of mutants that specifically lack Nrp1 in vessel endothelium (Fig. $2 \mathrm{M}, \mathrm{N}$ ) and that have vascular defects similar to the full Nrp1 knockout (Gu et al. 2003). We therefore conclude that normal vessel networks are not essential for the guidance of migrating facial branchiomotor somata.

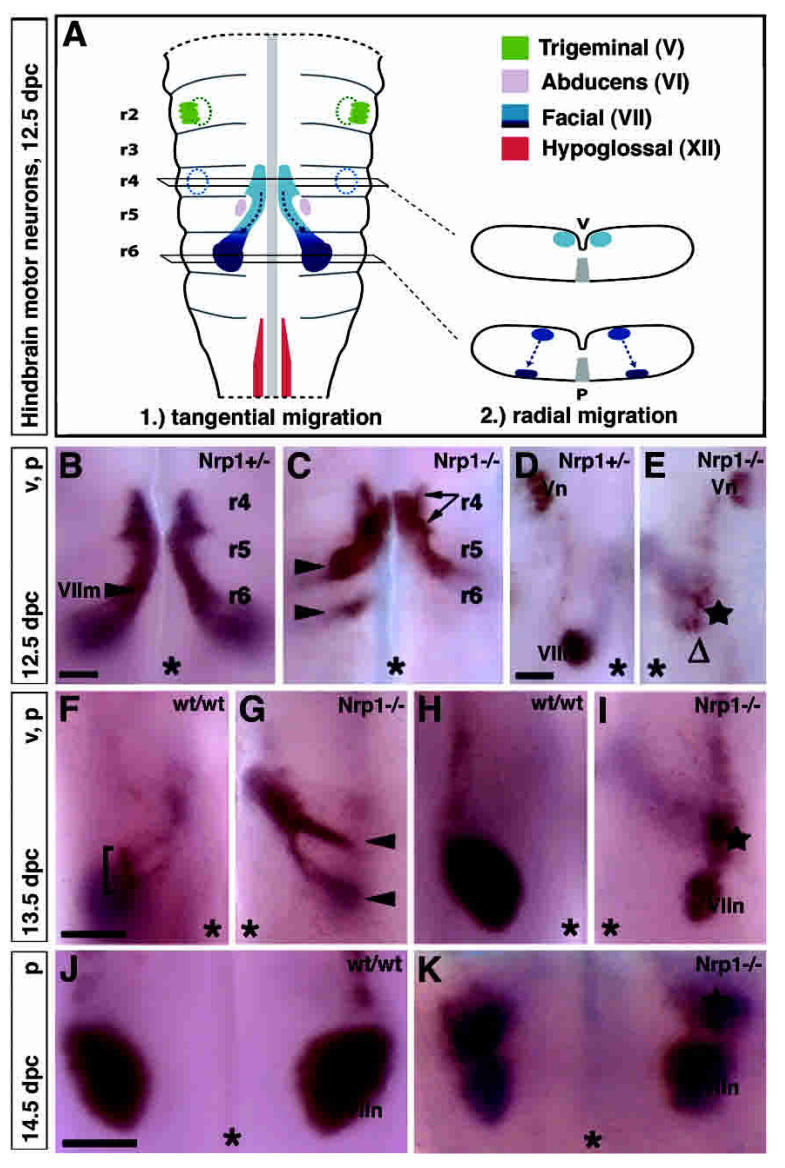




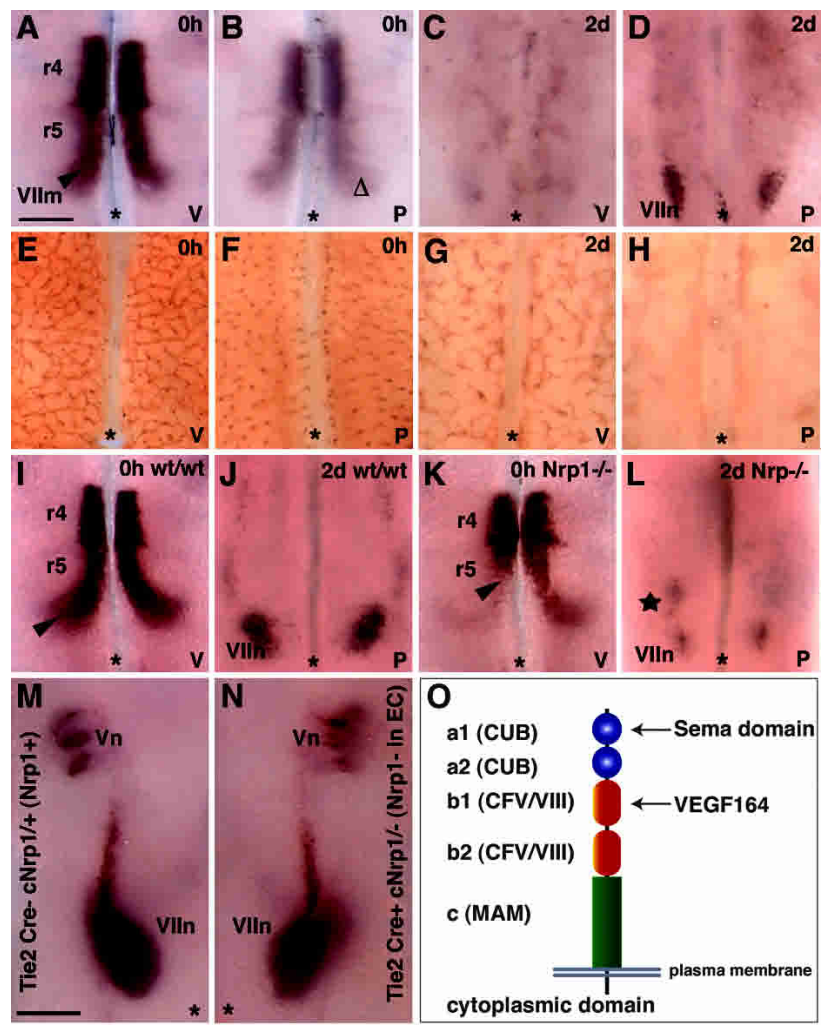

Figure 2. Soma migration of facial branchiomotor neurons does not rely on peripheral axons or normal vessel networks. $(A-D)$ Wild-type hindbrains $(11.5 \mathrm{dpc})$ were dissected free from peripheral axons and mesenchyme and fixed $(0 \mathrm{~h})$ or cultured for $2 \mathrm{~d}(2 \mathrm{~d})$ under serum-free conditions. In such explants, the somata of Isl1-positive facial branchiomotor neurons (VIIm, indicated with an arrowhead) traveled from the ventricular (v) side to form facial motor nuclei (VIIn) in a posterior position on the pial (p) side. Facial branchiomotor neurons were not seen prior to explanting on the pial side $(\Delta ; B)$, or on the ventricular side after $2 \mathrm{~d}$ in culture $(C)$. $(E-H)$ PECAM-positive vessel sprouts had entered the hindbrain from the pial side $(F)$ and formed an extensive vessel network on the ventricular side $(E)$ at the time of explanting $(0 \mathrm{~h})$. After $2 \mathrm{~d}$ in culture, vessel segments had severely degenerated on the ventricular side $(G)$ and completely degenerated on the pial side $(H)$. $(I-L)$ When 11.5-dpc littermate hindbrains containing $(I)$ or lacking $(K)$ Nrp1 were explanted, facial motor nuclei formed on the pial side in wild-type (VIIn; $J$ ) and mutant (VIIn; L) explants, but mutant explants also formed an ectopic anterior nucleus (star; $L$ ). As soma migration was delayed in explants lacking Nrp1, facial nuclei were never completely assembled after $2 \mathrm{~d}$ in culture, and they were therefore smaller than those in wild-type explants. $(M, N)$ When a conditionally targeted Nrp1 allele was removed from vascular endothelial cells (EC) with Cre recombinase under the control of the Tie2 promoter, the migration of facial branchiomotor somata was not impaired. One side of a control hindbrain $(M)$ and the opposite side of a stage-matched mutant hindbrain $(N)$ are shown next to each other to highlight the similar position of the facial (VIIn) relative to the trigeminal (Vn) nuclei. (O) Nrp1 contains CUB domains essential for binding the Sema domain of class 3 semaphorins and CFV/VIII domains for binding VEGF164. Bars, $250 \mu \mathrm{m}$ (one for $A-L$; one for $M, N$ ). The midline is indicated with an asterisk.
As the abnormal soma migration of facial branchiomotor neurons in the absence of Nrpl was not likely secondary to axon defects or disrupted vessel networks, we asked if it instead was caused by an inability of the somata to respond to a secreted Nrp1 ligand. Nrp1 binds with high affinity to several class 3 semaphorins, but also to an isoform of VEGF, termed VEGF165 in humans and VEGF164 in the mouse (Fig. 2O; He and TessierLavigne 1997; Kolodkin et al. 1997; Soker et al. 1998).

\section{Sema3A and Sema3C expression patterns are consistent with a role in the development of facial branchiomotor neurons}

Nrp1 serves as a high affinity receptor for several class 3 semaphorins, most notably Sema3A and Sema3C, and facial motor axons respond to Sema3A in culture models (Varela-Echavarria et al. 1997; for review, see Raper 2000). We found that Sema3A and Sema3C, but not Sema3B or Sema3E, were expressed strongly in the hindbrain (Fig. 3; data not shown). Whereas Sema3A was expressed more strongly in the environment (Fig. 3C, arrow), Sema3C was present at higher levels in the migrating neurons (Fig. 3E, arrowhead). At $12.5 \mathrm{dpc}$, Sema3A expression extended in a stripe throughout the length of the hindbrain, which coincided with the position where facial branchiomotor somata begin their descent through the basal plate (Fig. 3C, arrow). This observation raises the possibility that Sema3A provides a repulsive boundary that forces these neurons onto a new course. Interestingly, Sema3C expression was down-regulated once the facial branchiomotor somata had reached the pial surface (Fig. 3F, $\Delta$ ), suggesting that Sema3C promoted their migration, but did not play a role in motor nucleus assembly.

\section{Sema3A and Sema3C are not essential to guide facial motor neuron migration}

Although Sema3A was expressed in a pattern consistent with a role in neuronal guidance in the hindbrain, it was not required for the pathfinding of facial branchiomotor somata. Isl1-staining showed that the somata migrated normally on the ventricular side and arrived on the pial side in their proper location and at the appropriate time in the absence of Sema3A in all four cases examined (Fig. 3, cf. G and $\mathrm{H}_{\text {; }}$ data not shown). To address whether Sema3C was required for soma migration, we examined four mutants lacking this protein (Feiner et al. 2001), but found that Sema3C was also dispensable for the pathfinding of facial branchiomotor somata to their destination on the pial side of the hindbrain (Fig. 3, cf. G and I).

The lack of a phenotype in Sema3A or Sema3C knockouts may be explained by their functional redundancy during soma migration. To address this possibility, we examined compound mutants lacking both Sema3A and Sema3C and found that the soma migration of facial branchiomotor neurons occurred normally in all four 


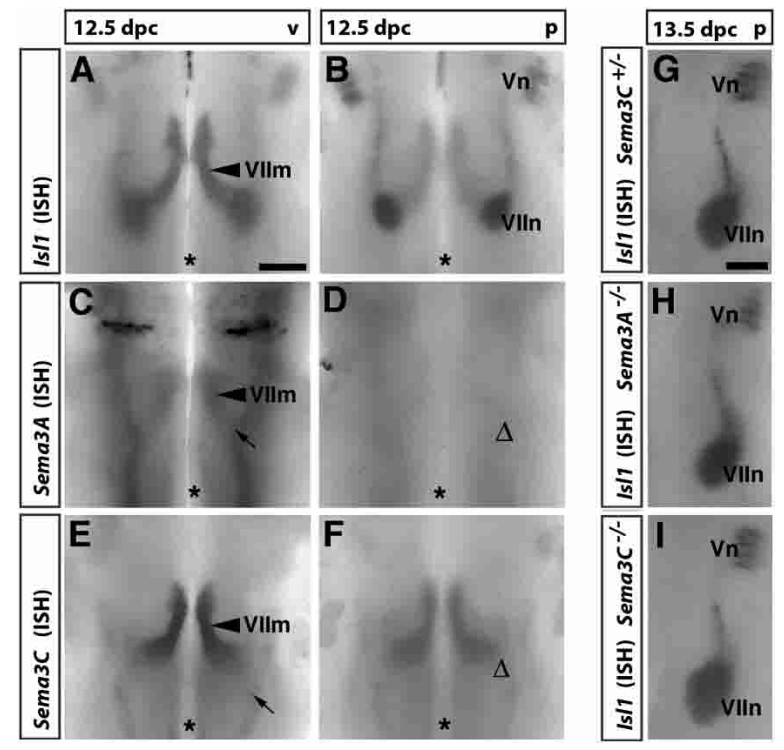

Figure 3. Sema3A and Sema3C are expressed in the hindbrain, but are not required for the pathfinding of facial branchiomotor somata. $(A, B)$ In situ hybridization (ISH) shows that at $12.5 \mathrm{dpc}$, the Is11-positive somata of facial branchiomotor neurons were migrating caudally (VIIm) on the ventricular side $(A)$ and began to condense into the paired facial motor nuclei (VIIn) on the pial side $(B)$ of the hindbrain. $(C-F)$ In stage-matched hindbrains, Sema3A $(C)$ and Sema3C $(E)$ were expressed on the ventricular side in the area where facial somata migrate (arrowheads in $C, E$ ) and in neighboring regions (arrows in $C, E)$. Sema3A $(D)$ and Sema3C $(F)$ were down-regulated on the pial side (cf. positions of $\Delta$ in $D, F$ and VIIn in $B) .(G-I)$ By $13.5 \mathrm{dpc}$, most facial somata were located within their nuclei (VIIn) on the pial side (cf. the size of facial motor nuclei in $B$ and $G)$. Loss of Sema3A $(H)$ or Sema3C $(I)$ did not impair the formation or positioning of facial motor nuclei (cf. the relative positions of VIIn and Vn). Bars: $A-F, 250 \mu \mathrm{m} ; G-I, 200 \mu \mathrm{m}$. The midline is indicated with an asterisk.

mutants examined (Fig. 4A-F). We then extended our analysis to $N r p 1^{\text {sema-l- }}$ mutants, which carry point mutations that abolish binding to the Sema domain of all class 3 semaphorins (Gu et al. 2002). Soma migration occurred normally in all five cases examined (Fig. 4G-L), suggesting that signaling of class 3 semaphorins through Nrp1 was not required for this process. Moreover, the observation that soma migration was not compromised in mutants with axon guidance defects, such as the Sema3A knockouts or Nrp $1^{\text {sema-/- }}$ mutants (Taniguchi et al. 1997; Gu et al. 2003), provides further support for the hypothesis that soma guidance is regulated independently of axon guidance.

Because semaphorin signaling through Nrp1 was not required to guide the migration of facial branchiomotor somata, we next asked if soma guidance relied on the presence of the alternative Nrp1 ligand VEGF164. Importantly, VEGF-mediated Nrp1 functions should be left intact when the Sema-binding domain of Nrp1 is mutated, as VEGF164 binding relies on a distinct region of Nrp1 (Fig. 2O; Gu et al. 2002; Mamluk et al. 2002).

\section{VEGF164 directs the soma migration of facial branchiomotor neurons}

Between 10.5 and $12.5 \mathrm{dpc}$, VEGF is expressed more abundantly on the ventricular relative to the pial side of the hindbrain to guide angiogenic sprouting from the perineural plexus toward the subventricular zone (Breier et al. 1992; Miquerol et al. 1999). To relate the expression pattern of VEGF to the development of facial branchiomotor neurons, we performed whole-mount in situ hybridization at $12.5 \mathrm{dpc}$ and found that the expression of the VEGF gene (VEGF-A) was up-regulated in the paired regions where facial motor nuclei formed (Fig. 5, cf. A and B). However, VEGF mRNA is unstable under normoxic conditions (e.g., Levy et al. 1995), and for this reason, its expression pattern is difficult to follow with the whole-mount in situ hybridization technique. To increase sensitivity and spatial resolution, we therefore monitored VEGF expression in subsequent experiments with a previously characterized $\beta$-galactosidase reporter, which carries a $L a c Z$ transgene in the $3^{\prime}$-UTR of VEGF-A (Miquerol et al. 1999).

As described before, we found that the Lac $Z$ reporter for $V E G F-A$ was initially expressed throughout the ventricular aspect of the hindbrain, but became down-regulated by $11.5 \mathrm{dpc}$, correlating with the formation of an extensive vascular plexus (Ruhrberg et al. 2002). However, expression in the midline area remained high at 12.5 and 13.5 dpc (Fig. 5D, arrow; data not shown). In addition, VEGF-A expression at $12.5 \mathrm{dpc}$ was elevated in paired dorso-medial stripes that extended throughout the length of the hindbrain (these paired stripes appear laterally in flat-mounted hindbrain preparations and are indicated by a curved arrow in Fig. 5D). The position of both these domains is consistent with a role as stepping stones for the somata along their journey: The somata first travel caudally in close proximity to the VEGF-rich midline and then change direction to travel toward the dorso-medial VEGF domains. However, neither the ventral nor the dorso-medial stripes of VEGF are restricted to the rhombomeres that contain the migrating somata of facial branchiomotor neurons. We therefore have to postulate the existence of cooperating cues, which prevent entry into anterior territories and help the migrating somata to change course once they arrive in $r 6$ territory, where they dive through the basal plate toward the site of facial nucleus assembly (see above). Importantly, the $L a c Z$ reporter confirmed that VEGF-A was expressed at $12.5 \mathrm{dpc}$ in the region where the facial nuclei formed (Fig. 5C,E), consistent with a role for VEGF in guiding the movement of the somata through the basal plate. At $13.5 \mathrm{dpc}, V E G F-A$ expression on the pial side resolved into a domain corresponding to the facial nucleus assembly site and an adjacent stripe (Fig. 5, cf. E and F).

VEGF is made in several isoforms, but VEGF164 is the only Nrp1-binding isoform expressed in the hindbrain during the time of facial branchiomotor neuron migration: VEGF188 is not expressed at significant levels at $12.5 \mathrm{dpc}$ (Ruhrberg et al. 2002), and VEGF120 (Soker et 


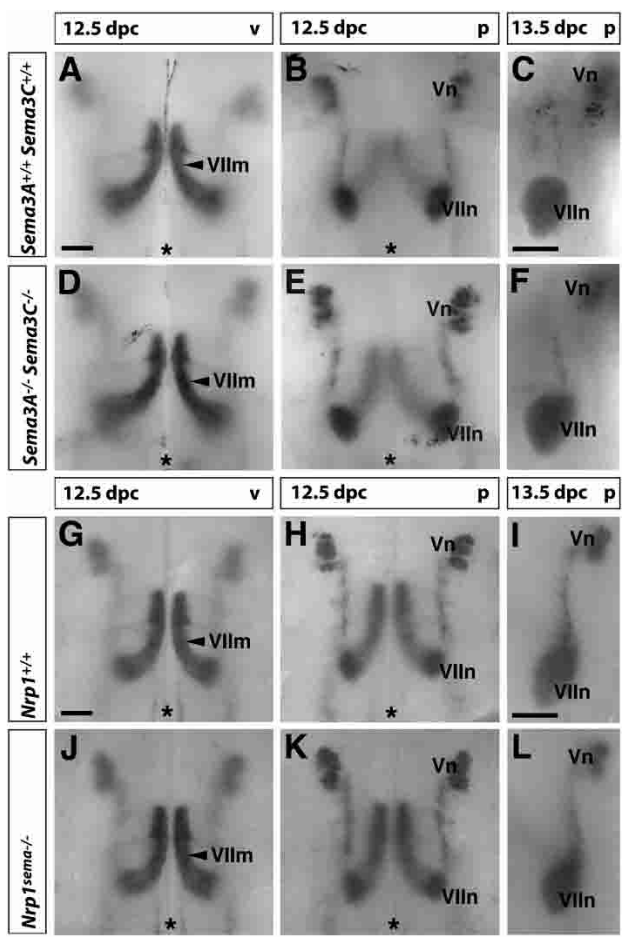

Figure 4. Semaphorin signaling through Nrp1 is not required for the migration of facial branchiomotor somata. $(A-F)$ Loss of both Sema3A and Sema3C did not impair the pathfinding of Isl1-positive facial somata on the ventricular side (cf. $A$ and $D$ ), nor the positioning of facial motor nuclei on the pial side at 12.5 dpc (cf. $B$ and $E$ ) or 13.5 dpc (cf. $C$ and $F$ ). $(G-L)$ Mutation of the Sema-binding domain of Nrpl did not impair pathfinding of Isl1-positive facial somata on the ventricular side (cf. $G$ and $J$ ), or the positioning of facial motor nuclei on the pial side at 12.5 dpc (cf. $H$ and $K$ ) or $13.5 \mathrm{dpc}$ (cf. $I$ and $L$ ). Bars, $250 \mu \mathrm{m}$ (one for $A, B, D, E$; one for $G, H, J, K$ ); $200 \mu \mathrm{m}$ (one for $C, F$; one for $I, L$ ). The midline is indicated with an asterisk.

al. 1998) and VEGF144 do not bind Nrp1 (Gluzman-Poltorak et al. 2000). Consistent with the idea that the Nrp1 ligand VEGF164 normally supports the soma migration of facial branchiomotor neurons in vivo, we found that mice genetically engineered to express VEGF120 at the expense of VEGF164 (VEGF ${ }^{120 / 120}$ mutants) (Carmeliet et al. 1999; Ruhrberg et al. 2002) displayed a spectrum of pathfinding errors similar to $\mathrm{Nrp}^{-/-}$mutants (cf. Figs. 1 and 5). In 9/9 cases, a large proportion of caudally migrating somata of facial branchiomotor neurons prematurely turned dorsally and dived through the basal plate in ectopic anterior locations (Fig. 5, cf. arrowheads in G and $\mathrm{H}$ ). As a result of this abnormal migration, the facial motor nuclei of $V E G F^{120 / 120}$ mutants appeared elongated (Fig. 5J) or dumbbell shaped (Fig. 5N). In 7/9 cases, one or both facial motor nuclei were shifted anteriorly altogether (Fig. 5, cf. I and J, K and L, M and N), and in 4/9 cases, facial somata scattered over an unusually large area on the pial side (Fig. 5, cf. boxed areas in $\mathrm{K}$ and L).

In contrast to hindbrains lacking VEGF164 $\left(V E G F^{120 / 120}\right), 8 / 8$ hindbrains expressing VEGF164 at the expense of all other isoforms (VEGF ${ }^{164 / 164}$ ) (Stalmans et al. 2002) showed no defects in the pathfinding of migrating facial somata and therefore assembled normal facial motor nuclei (Fig. 5O,P; data not shown). We therefore conclude that VEGF164 is the VEGF isoform that controls the soma migration of facial branchiomotor neurons.

To examine if VEGF164 controlled the migration of facial branchiomotor somata in the absence of functional axons, we added neutralizing antibodies specific for VEGF to the culture medium of hindbrain explants at 11.25 dpc (Fig. 5Q-S). We found that treatment with VEGF-neutralizing antibodies, but not treatment with control antibodies, caused the somata to prematurely turn dorsally in $\mathrm{r} 4$ or $\mathrm{r} 5$ in 5/6 wild-type explants and in 5/5 explants expressing VEGF164 only (Fig. 5R,S; data not shown).

Because VEGF is a chemoattractant for various cell types, including neuronal precursors (Zhang et al. 2003), it may also act as an attractant for migrating somata when presented in a localized fashion. To address this possibility, we implanted VEGF164-coated heparin beads into normal hindbrain tissue, and found that they promoted the migration of facial somata toward the VEGF164 source in 5/6 cases (Fig. 5U). In contrast, control beads without VEGF did not attract the migrating somata (4/4 cases) (Fig. 5T).

\section{Defective soma migration in the absence of Nrp1 and VEGF164 is not caused by abnormal hindbrain segmentation}

In several mouse mutants, the irregular soma migration of facial branchiomotor neurons can be attributed to defects in hindbrain segmentation (for review, see Cordes 2001). To examine if migration defects in $\mathrm{Nrp1}^{-/-}$and $V E G F^{120 / 120}$ mutants were an indirect consequence of an abnormal hindbrain structure, we used a marker that detects trigeminal and facial branchiomotor neurons as well as segment-specific neurogenesis patterns, the paired homeobox gene Phox2b (Davenne et al. 1999; Pattyn et al. 2000; Gavalas et al. 2003). At 12.5 dpc, Phox2b is expressed in the wild-type hindbrain in both migrating facial branchiomotor somata and in three neuron columns that reflect the segmental organization of the hindbrain (Supplementary Fig. S2A,B; Gavalas et al. 2003). Using this marker, we confirmed the abnormal soma migration of facial branchiomotor neurons in $4 / 4$ $N r p 1^{-/-}$and 2/2 VEGF $F^{120 / 120}$ mutants, but found that other Phox $2 b$-positive neurons were organized into their appropriate columns (Supplementary Fig. S2C-F). These observations suggest that the abnormal soma migration of facial branchiomotor neurons in the absence of Nrp1 or VEGF164 is not due to a defect in hindbrain segmentation.

\section{Defective soma migration of facial branchiomotor neurons in the absence of VEGF164 is not accompanied by axon guidance defects in the periphery}

Loss of Nrp1 or Sema3A results in the defasciculation of several cranial nerves, including the trigeminal and fa- 
Schwarz et al.

Figure 5. VEGF controls the pathfinding of facial branchiomotor somata. $(A, B)$ Whole-mount in situ hybridization at $12.5 \mathrm{dpc}$ shows expression of the $V E G F-A$ gene $(B)$ in the area were the Isl1-positive somata of facial branchiomotor neurons assemble into facial motor nuclei (VIIn) on the pial side of the hindbrain $(A)$. $(C-F)$ Expression of a VEGF-A LacZ reporter at $12.5(C-E)$ and $13.5 \mathrm{dpc}(F) .(C)$ At 12.5 dpc on the pial side, VEGF-A LacZ expression was prominent in the area of facial motor nucleus assembly (VIIn). (D) At $12.5 \mathrm{dpc}$ on the ventricular side, VEGF-A LacZ expression was prominent near the midline (arrow), in a more dorsally located pair of stripes (wavy arrow), and in the area where the hypoglossal nuclei form (XIIn). (E) A higher magnification of the boxed area in $C$. $(F)$ At $13.5 \mathrm{dpc}$, expression was elevated in the area of facial motor nucleus assembly and in an adjacent stripe (open arrowhead). $(G-N)$ The migration of the Isl1-positive somata of facial branchiomotor neurons (arrowheads) was observed in the presence (wt/wt or wt/ $120)$ or absence $(120 / 120)$ of VEGF164. In the absence of VEGF164, facial branchiomotor neurons formed nuclei in anterior positions, which appeared elongated or dumbbell shaped (stars; $J, L, N)$. One side of a control hindbrain and the opposite side of a stage-matched mutant hindbrain are shown next to each other in $G-L$ to highlight the position of the facial branchiomotor neurons relative to the hypoglossal (XIIn; $G, H)$ or trigeminal $(V n ; I-L)$ nuclei (cf. the length of the square brackets in $G-J$ controls and mutants). In some mutants, somata emerged in an abnormally large area on the pial side and could be identified as scattered Is11-positive cells (cf. boxed
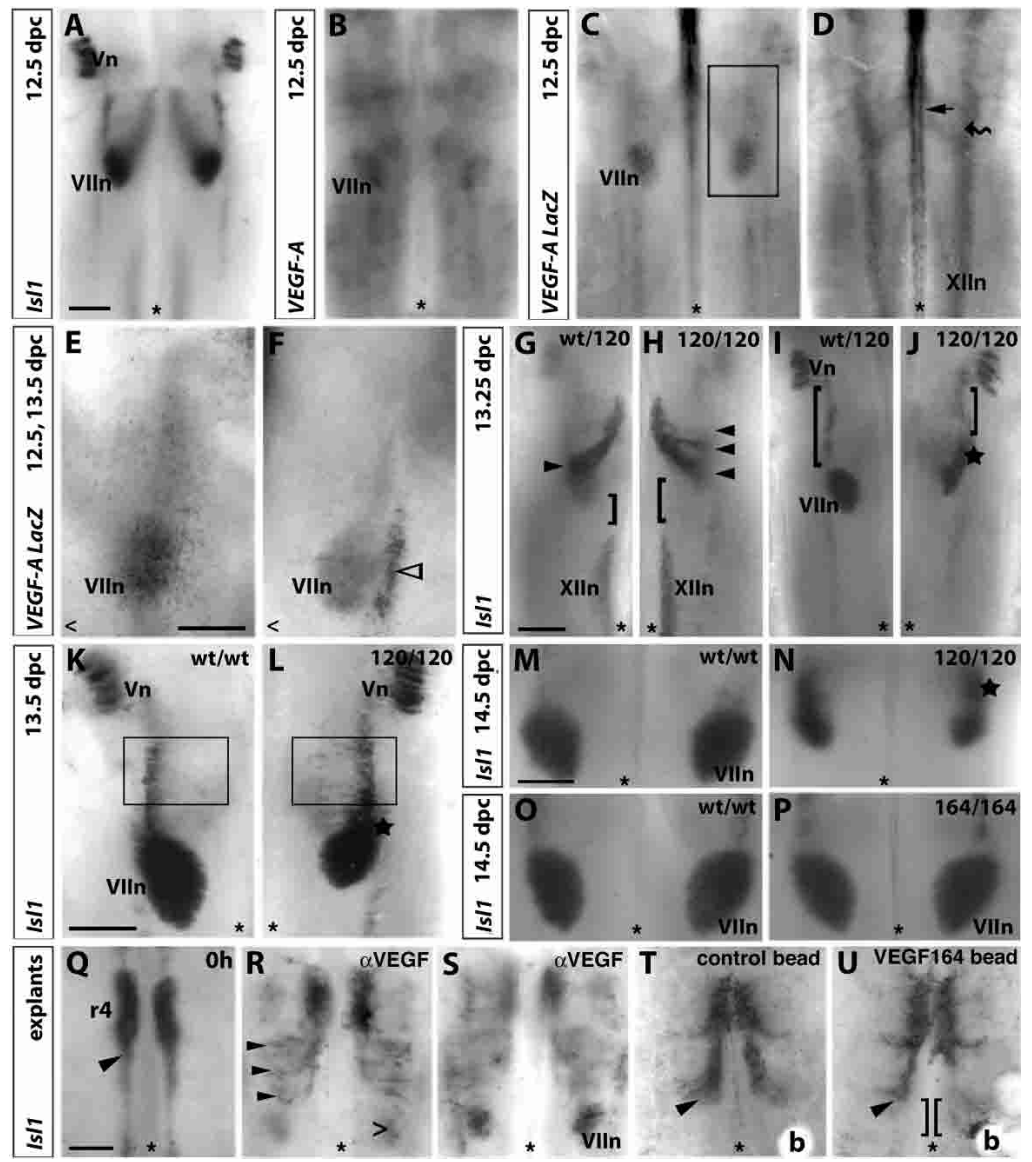
areas in $K$ and $L) .(O, P)$ A comparison of the facial motor nuclei (VIIn) formed in littermate hindbrains capable of expressing all VEGF isoforms (wt/wt; O) or VEGF164 only (164/164; $P$ ) showed that VEGF164 was sufficient to drive normal soma migration. (Q-U) Hindbrain tissue (11.25 dpc) expressing VEGF164 only was fixed $(0 \mathrm{~h} ; \mathrm{Q})$ or cultured for $2 \mathrm{~d}$ in the presence of function-blocking antibodies for VEGF $(\alpha$ VEGF; $R, S)$ to ablate VEGF164 function. When VEGF164 function was blocked, somata continued to migrate caudally out of r4 on the ventricular side, and a proportion of somata dived through the basal plate in the expected position (open arrowhead; $R$ ) to form facial motor nuclei on the pial side (VIIn; $S$ ). However, ectopically migrating somata were seen on the ventricular side in the r 5 territory $(\operatorname{arrowheads}$ in $R)$. $(T, U)$ When heparin beads (b) were implanted into normal hindbrain tissue, somata did not respond to control beads $(T)$, but moved toward beads coated with VEGF164 $(U)_{\text {; }}$ accordingly, somata were positioned more posteriorly on the side containing VEGF beads relative to the untreated hindbrain side (double bracket in $U$ ). Bars, $250 \mu \mathrm{m}$ (one for $A-D$; one for $E, F$ one for $G-J$; one for $K, L$; on for $M-P$; one for $Q-U)$. The midline is indicated with an asterisk; in $K$ and $L$ the midline is located outside the panel, but its position is indicated with an open arrowhead.

cial nerves (Fig. 6B; Kitsukawa et al. 1997; Taniguchi et al. 1997), and VEGF164 has been hypothesized to provide a positive signal for axon guidance (Carmeliet 2003). We therefore asked if loss of VEGF164 caused facial nerve axons to behave abnormally. We found that loss of VEGF164 did not impair target finding in the periphery, as all major branches of the facial nerve could be identified in their appropriate position in 5/5 cases examined (Fig. 6A). Moreover, unlike mutants lacking Nrp1 or Sema3A, mutants lacking VEGF164 did not show defasciculation of the facial nerve (Fig. 6, cf. B and D). Taken together, these observations support the idea that defects in soma guidance are not secondary to axon guidance defects. Rather, soma migration and axon behavior of facial branchiomotor neurons are regulated independently by the two Nrp1 ligands VEGF164 and Sema3A (Fig. 6E).

\section{Discussion}

\section{VEGF164 and Nrp1 guide soma migration in the hindbrain}

To address if Nrp1 controlled soma and axon migration of the same neurons, we focused on facial branchiomotor neurons; firstly, because their axons require Nrp1 for their guidance, and secondly, because their somata undergo an extensive migration through several hindbrain segments that is readily monitored (Fig. 1A; Supplementary Fig. S1). Using two markers for developing facial branchiomotor neurons, Isl1 and Phox2b, we found that loss of Nrp1 impaired soma pathfinding during their tangential, but not their radial migration phase, and therefore caused the formation of misshapen or ectopic facial (VIIth) motor nuclei (Fig. 1; Supplementary Fig. S2). 


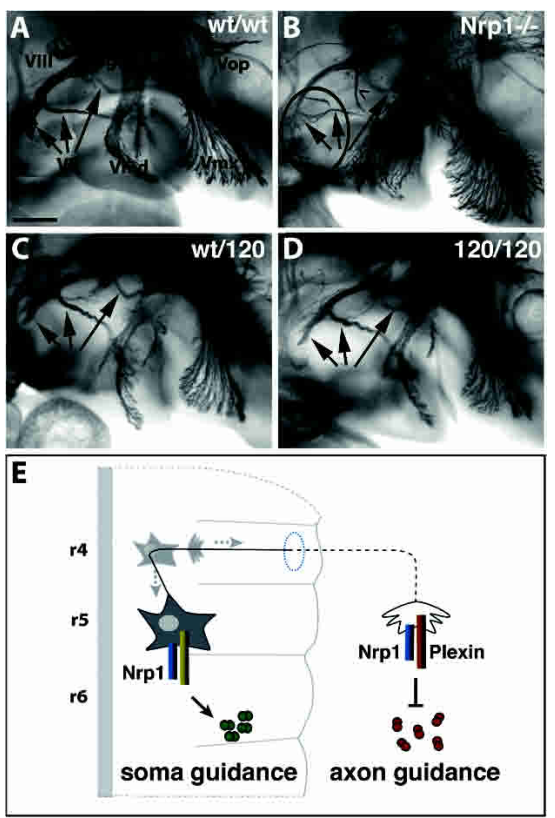

Figure 6. VEGF164 is not required for the target finding of facial nerve axons. $(A-D)$ Neurofilament staining of cranial nerve axons in 11.5-dpc embryos. Facial nerve axons extend normally into the branchial arches in the presence $(A, C)$ or absence $(D)$ of VEGF164, but they defasciculate in mutants lacking Nrp1 $(B)$. The three major branches of the lower facial nerve (VII) are indicated with arrows. The position of the maxillary (Vmx), mandibular (Vmd), and ophthalmic (Vop) branches of the trigeminal nerve, the vestibulocochelar nerve (VIII) and the trigeminal (Vg) and facial (VIIg) ganglia are indicated in $A$. In $B$, defasciculation of the facial nerve is highlighted with a circle, and an abnormally positioned branch of the facial nerve is indicated with an open arrowhead. The trigeminal nerve branches are also defasciculated. Bar, $250 \mu \mathrm{m}$. (E) Working model for the control of axon and soma guidance in facial branchiomotor neurons by Nrp1 ligands. Sema3A binds to Nrp1-containing receptors in the growth cone to control axon guidance in the periphery, while VEGF164 binds to Nrp1-containing receptors on the cell body in the hindbrain to control soma migration. The most prominent site of VEGF164 expression on the pial hindbrain side is shown in green to indicate its likely role as an attractive signal for migrating somata. Sema3A is shown in red to indicate its role as an axon repellent in the periphery. Nrp1 associates with plexins to transmit semaphorin signals in the growth cone, but the signaling coreceptor in the soma is not known.

As loss of Nrp1 affects axon pathfinding in the periphery and compromises vascularization of the neural tube, we first examined if the defective soma migration of facial branchiomotor neurons was secondary to axonal or vascular patterning defects. However, there was no relationship between the control of soma and axon pathfinding, and proper vessel patterning was not a prerequisite for normal soma migration (Figs. 2-4). These observations suggested that Nrpl was required cell-autonomously to mediate the response of migrating somata to a secreted Nrp1 ligand.

To determine which Nrp1 ligands control the behavior of facial branchiomotor somata, we analyzed the expres- sion patterns and functional requirements for class 3 semaphorins and VEGF. Based on their expression patterns and functions in vitro, Sema3A and Sema3F have both been implicated in the Nrp1/Nrp2-mediated control of interneuron migration in the telencephalon (Marin et al. 2001; Tamamaki et al. 2003). The observation that neither Sema3A nor Sema3C, nor any other Nrp1binding semaphorin, was required for the Nrp1-mediated pathfinding of facial branchiomotor neurons was therefore unexpected (Figs. 3, 4). Even more surprising was the finding that soma behavior of these neurons instead relied on the alternative Nrp1 ligand VEGF164, which is better known for its role in supporting the migration of cultured endothelial cells (Soker et al. 1998).

The lack of a soma migration defect in mutants lacking semaphorin signaling though Nrp1 may be due to compensation by Nrp2-based semaphorin signaling, as Nrp2 is expressed in migrating facial somata (Q. Schwarz and C. Ruhrberg, unpubl.). However, we believe that this possibility is unlikely, because loss of Nrp2 or its ligands Sema3C or Sema3F did not obviously impair the soma migration of facial branchiomotor neurons (Fig. 3; Q. Schwarz, V. Vivancos, M. Studer, S. Guthrie, C. Gu, A. Sahay, A. Kolodkin, D. Ginty, and C. Ruhrberg, unpubl.). We therefore conclude that semaphorins at best provide a backup system for VEGF164 signals. Such a function may only be revealed in compound mutants, for example, those carrying Sema3A knockout alleles on a VEGF120 background. Alternatively, the striking expression patterns of Sema3A and Sema3C in the hindbrain may not indicate a role in soma guidance, but instead reflect other functions during hindbrain patterning. For example, Sema3A may provide repulsive boundaries for axon guidance within the hindbrain, and Sema3C may be expressed by migrating neurons to repel Nrp1/Nrp2bearing vascular endothelial cells in the neuronal migration route.

Whereas semaphorin signaling was dispensable, VEGF164 was necessary both in vitro and in vivo for the correct pathfinding of facial branchiomotor somata (cf. Figs. 4 and 5). The soma migration defect was fully penetrant in mutants lacking VEGF164, and they displayed a similar spectrum of pathfinding errors to Nrp1 mutants, in agreement with biochemical data that demonstrate the ability of VEGF164 to bind to Nrp1 (Soker et al. 1998). The combined analysis of hindbrains lacking VEGF164 (VEGF $\left.{ }^{120 / 120}\right)$ or expressing VEGF164 at the expense of all other isoforms (VEGF $\left.F^{164 / 164}\right)$ shows that VEGF164 is the only VEGF isoform that is both necessary and sufficient for the soma migration of facial branchiomotor neurons in vivo (Fig. 5). As VEGF188 is not expressed at significant levels in the hindbrain during the time of facial branchiomotor neuron migration (Ruhrberg et al. 2002), it cannot normally contribute to facial soma pathfinding. However, VEGF188 does contain the domain that mediates Nrpl binding in VEGF164, and it will therefore be interesting to determine if the ectopic expression of VEGF188 in the developing hindbrain can rescue the soma migration defects caused by a lack of VEGF164. 
The ability of VEGF164-coated heparin beads to attract migrating facial branchiomotor somata in explant culture suggests that VEGF is a chemoattractant for Nrp1-expressing neurons (Fig. 5). In this assay, VEGF is presented in a localized form in conjunction with heparin, an important cofactor for Nrp1 binding (Gluzman-Poltorak et al. 2000). In contrast to heparin-rich VEGF beads, the addition of VEGF164 to the culture medium did not perturb soma migration (data not shown). This may be explained by a failure of exogenously added VEGF to be absorbed into the heparin sulfate proteoglycan (HSPG)-rich hindbrain matrix; for example, VEGF-binding sites may not be accessible or already saturated. Alternatively, VEGF may only act as a chemoattractant for migrating neurons when presented in a steep gradient, as observed in the case of migrating endothelial cells in the embryonic hindbrain and retina (Ruhrberg et al. 2002; Gerhardt et al. 2003).

Despite the striking similarity in pathfinding errors, there were some differences in the migratory behavior of facial branchiomotor somata in $N r p 1^{-1-}$ and $V E G F^{120 / 120}$ mutants. For example, facial somata split into several distinct streams in $\mathrm{Nrp1}^{-/-}$mutants, but they spread out more in VEGF $F^{120 / 120}$ mutants (cf. Figs. 1 and 5). This difference may relate to Nrp1's ability to interact with partners other than VEGF164, for example, the cell adhesion molecule L1 (Castellani and Rougon 2002) or the Nrp2 receptor (Gluzman-Poltorak et al. 2000). A second reason for the differences between the soma migration defects of $\mathrm{Nrp1}^{-/-}$and VEGF ${ }^{120 / 120}$ mutants may relate to VEGF164's ability to participate both in isoformspecific signaling and in the formation of matrixassociated VEGF concentration gradients. VEGF $F^{120 / 120}$ mutants produce excess amounts of VEGF120 at the expense of VEGF164, and this increase in soluble over heparin-binding isoforms promotes the dispersion of VEGF in the hindbrain, and therefore in the environment of migrating somata (Ruhrberg et al. 2002). As developing motor neurons express Flt1 (Q. Schwarz, M. Golding, and C. Ruhrberg, unpubl.), they may have the capacity to detect ectopic VEGF deposits composed solely of VEGF120. A third possibility for the differences between the soma migration defects of $\mathrm{Nrp1}^{-/-}$and $V E G F^{120 / 120}$ mutants may relate to the observation that VEGF164 and VEGF144 can both bind to Nrp2 (Gluzman-Poltorak et al. 2000). Because Nrp2 is expressed by the migrating facial somata (see above), it is conceivable that VEGF144/164 signaling through Nrp2 could partially compensate for loss of VEGF164 signaling in $\mathrm{Nrp1}^{-/-}$mutants. In contrast, VEGF $F^{120 / 120}$ mutants lack both VEGF144 and VEGF164, and therefore loss of VEGF164 signaling through Nrp1 could not be rescued by VEGF144/164 signaling through Nrp2. As full Nrp1/Nrp2 double knockouts are lethal early during embryonic development (Takashima et al. 2002), it would be necessary to generate conditional double knockouts to test the hypothesis that Nrp2 can compensate for Nrp1 signaling in facial branchiomotor neurons.
Independent control of soma migration and axon guidance through Nrp1 ligands: cooperation, not competition of VEGF164 and Sema3A

It has previously been suggested that axon guidance can occur independently of signals from the soma, because axons of retinal ganglion cells continue to navigate after removal of their somata (Harris et al. 1987). We now show that the reverse is also true, as soma pathfinding of facial branchiomotor neurons continues normally after axons have been removed in explant culture (Fig. 2). The analysis of several different mouse mutants also supports the idea that soma migration and axon guidance of the developing facial nerve are regulated independently. Firstly, loss of HoxB1 or the zebrafish trilobite mutation, like loss of VEGF164, disrupt the soma migration of facial branchiomotor neurons without affecting the target finding of facial nerve axons (Fig. 6; Studer et al. 1996; Bingham et al. 2002; Gavalas et al. 2003). Secondly, loss of Sema3A disturbs the behavior of facial nerve axons (Taniguchi et al. 1997), but does not perturb the soma migration of facial branchiomotor neurons (Fig. 3).

Because VEGF164 controls the soma positioning of facial branchiomotor neurons, but is not required for their axon guidance, and, conversely, Sema3A is essential for their axon guidance, but not the pathfinding of their somata, we conclude that Nrp1 supports soma and axon migration independently through the binding of two different ligands (Fig. 6E, model). Therefore, Sema3A and VEGF do not appear to compete with each other, but instead cooperate by patterning different compartments of the same cell.

The concept that Nrp1 ligands cooperate to pattern neurons is novel and differs from an earlier model of Nrp1 function in neurons, which suggests that the competition of Sema3A and VEGF164 for binding to Nrp1 on the axonal growth cone is critical to steer migrating axons (Carmeliet 2003). The competitive model for neuronal patterning by Nrp1 ligands was derived from work in several cell culture systems, where Sema3A modulates the activity of VEGF on vascular endothelial cells (Miao et al. 1999), while VEGF counters Sema3A-mediated responses of neural progenitor cells (Bagnard et al. 2001). Both models may not be mutually exclusive, but could operate in distinct situations. For example, the balance of VEGF164 and Sema3A may influence the decision of axons to grow in tight fascicles or defasciculate, or influence the patterning of growing blood vessel networks.

The ability of Nrp1 to participate in two separate patterning events of the same neuron through its alternative ligands raises the possibility that it exists in different conformational states (1) to influence Nrp1's preference for its structurally distinct ligands VEGF164 and Sema3A, and (2) to stimulate distinct intracellular signaling events in the soma and the axon. For example, Nrp1 may associate with a different signaling coreceptor in soma and axon, where Flk1 or Flt1 may mediate a VEGF response, while plexins would transduce a semaphorin signal. We were not able to detect Flk1 in devel- 
oping motor neurons between 10.5 and $14.5 \mathrm{dpc}$, consistent with the previous observation that Flk1 expression in neurons is not obviously required for brain development (Haigh et al. 2003). In contrast, we found expression of Flt1 and three A-type plexins in facial branchiomotor neurons by in situ hybridization (Q. Schwarz and C. Ruhrberg, unpubl.). One attractive hypothesis may therefore be that VEGF recruits Flt1 to Nrp1 to mediate an attractive response to VEGF in the soma, whereas Sema3A/Nrp1 recruits a plexin family member to induce repulsion of axons. Alternatively, VEGF164 and Sema3A may recruit different plexin family members to Nrp1. The hypothesis that Nrp1 requires different coreceptors to signal VEGF164 and Sema3A responses might be tested through a comparative analysis of soma and axon behavior in mouse mutants carrying conditional Flk1 or Flt1 knockout alleles or plexin lossof-function mutations.

\section{Relationship of VEGF 164/Nrp1 to other proteins implicated in neuronal migration}

In several mouse mutants, defective facial nerve development can be attributed to errors in the segmental patterning of the hindbrain (for review, see Cordes 2001). In some of these mutants, the somata of facial branchiomotor neurons encounter an abnormal environment during their migration, because transcription factors that control hindbrain segmentation are lost (e.g., kreisler). In another type of hindbrain patterning mutant, facial branchiomotor neurons undergo a switch in molecular identity together with their $\mathrm{r} 4$ environment, and their somata therefore assume a migratory path characteristic of trigeminal branchiomotor neurons (HoxB1 knockouts). However, the soma migration defects in $\mathrm{Nrp1}^{-/-}$and $V E G F^{120 / 120}$ mutants are not caused by errors in the segmental patterning of the hindbrain (Supplementary Fig. S2). In this respect, both mouse mutants resemble the zebrafish mutant trilobite, in which soma migration, but not hindbrain patterning or neuronal differentiation, is affected (Bingham et al. 2002). In these mutants without segmentation defects, abnormal neuronal migration is likely due to cell-autonomous defects, in which neurons fail to respond to environmental cues.

We have been able to demonstrate a role for VEGF164 as a guidance cue for Nrp1-expressing motor neurons using a combination of mouse genetics and explant cultures (Fig. 5). Moreover, we have preliminary evidence to suggest that VEGF164 and Nrp1 also contribute to the guidance of migrating inferior olive neurons (Q. Schwarz and C. Ruhrberg, unpubl.). However, just as Sema3A is only one of a large collection of axon guidance cues that act synergistically to control the pathfinding of peripheral nerves (for review, see Dickson 2002), VEGF164 is likely to act in concert with other guidance cues. In agreement with this idea, the secreted proteins reelin and netrin also play essential roles during the migration of facial branchiomotor and inferior olive neurons, but loss of either protein does not abolish soma pathfinding, as observed for loss of VEGF164 (Bloch-Gallego et al.
1999; Ohshima et al. 2002). We therefore anticipate that VEGF synergises with other guidance pathways to control the migration of several different neuron classes in the brain.

Similar to the loss of an environmental guidance cue, the mutation of genes that control cellular responses to such extracellular stimuli can cause neuronal migration defects. For example, the mutation of the intracellular proteins Dab1 or Cdk5 or the Cdk5-associated regulatory subunits p35 and p38 disrupts the soma migration of facial branchiomotor neurons (Ohshima et al. 2002). The VEGF/Nrp1 complex may operate in the same genetic pathway that contains Cdk5 and cytoplasmic dynein, because (1) Nrp1-containing receptor complexes can recruit Cdk5 (Sasaki et al. 2002), (2) Cdk5 can phosphorylate proteins that interact with dynein and have been implicated in nuclear translocation, and (3) loss of VEGF164/Nrp1 signaling and a dynein point mutation cause similar defects during facial branchiomotor soma migration (cf. Figs. 1, 5 shown here and Fig. 3 in Hafezparast et al. 2003). We therefore speculate that binding of VEGF164 to Nrp1 activates a signaling pathway, which modifies Cdk5 activity to control microtubule dynamics and thereby promotes nuclear and organelle translocation during soma migration.

\section{Potential roles for VEGF/Nrp1 signaling in neuronal diseases}

Because VEGF164 and Nrp1 play essential roles during neuronal migration in the central nervous system, defective VEGF signaling might contribute to human disease. Moebius syndrome (MBS, OMIM 157900) is an inborn human condition that affects the VIIth cranial nerve, and three chromosomal loci have been associated with familial Moebius syndrome. MBS1 maps to chromosome 13q12.2-q13, which harbors the Flt1 gene (OMIM 165070); MBS2 maps to 3q21-q22, which contains PlexinA1 (OMIM 601055) and two other genes whose products have the potential to interact with Nrp1, PlexinD1 (OMIM 604282), and Sema5B (LOCUS LINK ID 54437). It will therefore be interesting to determine if any of these genes are linked to the disease locus. Transient ischemia during hindbrain development may also contribute to Moebius syndrome (e.g., Verzijl et al. 1999). As VEGF-A gene expression is regulated by hypoxia, defective VEGF signaling might contribute to the aetiology of sporadic Moebius cases by simultaneously disturbing hindbrain vascularisation and facial nerve development.

Low levels of VEGF contribute to postnatal motor neuron degeneration in mice, in which motor neuron cell death has been attributed to decreased neuroprotection by VEGF or reduced vascular perfusion of motor nerves (Oosthuyse et al. 2001). In addition to this mechanism, our findings raise the possibility that motor nerves forming in the presence of low VEGF levels may already be compromised at birth and therefore predisposed to succumb to further insults during postnatal life. An association between abnormal motor neuron development 
and postnatal motor neuron degeneration has previously been demonstrated in the Loa mouse, which carries a missense mutation in the heavy chain of cytoplasmic dynein (Hafezparast et al. 2003). Moreover, low VEGF levels due to an inborn promoter polymorphism predispose humans to Amyotrophic Lateral Sclerosis, a fatal motor neuron disease (Lambrechts et al. 2003).

\section{Conclusion}

VEGF controls the migratory behavior of facial branchiomotor neurons in the developing brain in a mechanism that relies on the VEGF164 isoform and its receptor Nrp1. VEGF164 cooperates with the alternative Nrp1 ligand Sema3A to pattern different compartments of the same nerve, where VEGF164 is required only for soma guidance and Sema3A only for axon guidance. Altered VEGF expression during embryogenesis may therefore lead to neurological defects in addition to vascular deficiency.

\section{Materials and methods}

\section{Animals}

To obtain embryos of defined gestational ages, animals were mated in the evening, and the morning of vaginal plug formation was counted as $0.5 \mathrm{dpc}$. To stage-match embryos within a litter, or between litters from different matings, we compared somite numbers $(10.5 \mathrm{dpc})$ or facial and limb development (11.5-14.5 dpc). Nrp $1^{-/-}$mutants have been described previously (Kitsukawa et al. 1997). To obtain 14.5-dpc Nrp1 $1^{-/-}$mutants, heterozygous females in a CD1 background were mated with heterozygous males in the JF1 background (kindly provided by T. Shiroshi, National Institute of Genetics, Mishima, Japan). Nrp1 $1^{\text {sema-l- }}$ and endothelial-specific Nrp1 knockouts (Gu et al. 2003), Sema3A knockouts (Taniguchi et al. 1997), and Sema3C knockouts (Feiner et al. 2001) were maintained as described previously. Sema3C/Sema3A double knockouts were provided by J. Raper (University of Pennsylvania, Philadelphia, PA). $V E G F^{120 / 120}$ and VEGF ${ }^{164 / 164}$ mutants have been described previously (Carmeliet et al. 1999; Stalmans et al. 2002). Male mice containing the LacZ reporter in the 3'-UTR of one VEGF-A allele (Miquerol et al. 1999) were bred with wild-type CD1 females to produce heterozygous embryos for visualization of $\beta$-galactosidase activity.

In situ hybridization, immunohistochemistry, and visualization of $\beta$-galactosidase activity

Immunohistochemistry and visualization of $\beta$-galactosidase activity were performed as described previously (Ruhrberg et al. 2002; Hafezparast et al. 2003), with the exception that nerves were visualized with rabbit anti-neurofilament antibodies (Chemicon) followed by horseradish-peroxidase conjugated anti-rabbit antibodies (Dako) as described in Maina et al. (1997). For in situ hybridization, tissues were hybridized with digoxigenin-labeled probes specific for the following genes: Is11 (Ericson et al. 1992), Nrp1 (He and Tessier-Lavigne 1997), Sema3A and Sema3C (gifts of M. Tessier-Lavigne, Stanford University, Stanford, CA), Phox2b (Pattyn et al. 2000), and VEGF164 (Breier et al. 1992). In situ hybridization was performed as described in Hafezparast et al. (2003), with the exception that VEGF-A was detected using the method described in Riddle et al. (1993). Images were recorded using the MZ16 microscope (Leica) equipped with a ProgRes C14 digital camera (Jenoptiks) and processed using Openlab 2.2 software (Improvision Ltd.) and Adobe Photoshop 7.0 (Adobe Systems, Inc.).

\section{Explant cultures}

For explant cultures, hindbrains were dissected in cold L15 (Invitrogen) free from branchial arch tissue, axons, sensory ganglia, and pial membranes. Hindbrain tissue was cultured for 1 or $2 \mathrm{~d}$ on Costar culture inserts with an 8-micrometer pore size (Corning, Inc.) in Neurobasal medium containing B27 supplement (Invitrogen). In some experiments, explants were cultured in the presence of a function-blocking antiserum for VEGF (affinitypurified rabbit anti-mouse VEGF; Research Diagnostics, Inc.) or normal rabbit IgG (R\&D Systems). Affi-Gel heparin beads (BioRad Laboratories) were washed several times in PBS and soaked in VEGF164 (Preprotech Ltd.) overnight at $4^{\circ} \mathrm{C}$ prior to implantation into explants. Explants were processed for in situ hybridization or immunohistochemistry as described above.

\section{Acknowledgments}

We acknowledge with gratitude John Greenwood, Lynda Erskine, Matthew Golding, and Giampietro Schiavo for their support; Takeshi Yagi, Anthony Holtmaat, Toshihiko Shiroshi, and Jonathan Raper for providing mice; Marc Tessier-Lavigne, Thomas Jessel, Georg Breier, and Jean-Francois Brunet for providing cDNA probes; the Biological Resources Units at Clare Hall Laboratories and the Institute of Ophthalmology for help with animal husbandry; Fabrizia Cesca for help with genotyping; and Lynda Erskine, Glen Jeffrey, and Robb E. Krumlauf for helpful discussions. A.L.K. and D.D.G. are funded by the NIH (NIH MH59199). D.D.G. is an investigator of the Howard Hughes Medical Institute. Q.S. and C.R. are funded by an MRC Career Development Award to C.R.

\section{References}

Altman, J. and Bayer, S.A. 1982. Development of the cranial nerve ganglia and related nuclei in the rat. Adv. Anat. Embryol. Cell Biol. 74: 1-90.

Auclair, F., Valdes, N., and Marchand, R. 1996. Rhombomerespecific origin of branchial and visceral motoneurons of the facial nerve in the rat embryo. J. Comp. Neurol. 369: 451461.

Bagnard, D., Vaillant, C., Khuth, S.T., Dufay, N., Lohrum, M., Puschel, A.W., Belin, M.F., Bolz, J., and Thomasset, N. 2001. Semaphorin 3A-vascular endothelial growth factor-165 balance mediates migration and apoptosis of neural progenitor cells by the recruitment of shared receptor. I. Neurosci. 21: 3332-3341.

Bingham, S., Higashijima, S., Okamoto, H., and Chandrasekhar, A. 2002. The zebrafish trilobite gene is essential for tangential migration of branchiomotor neurons. Dev. Biol. 242: $149-160$.

Bloch-Gallego, E., Ezan, F., Tessier-Lavigne, M., and Sotelo, C. 1999. Floor plate and netrin-1 are involved in the migration and survival of inferior olivary neurons. I. Neurosci. 19: 4407-4420.

Breier, G., Albrecht, U., Sterrer, S., and Risau, W. 1992. Expression of vascular endothelial growth factor during embryonic angiogenesis and endothelial cell differentiation. Development 114: 521-532. 
Carmeliet, P. 2003. Blood vessels and nerves: Common signals, pathways and diseases. Nat. Rev. Genet. 4: 710-720.

Carmeliet, P., Ng, Y.S., Nuyens, D., Theilmeier, G., Brusselmans, K., Cornelissen, I., Ehler, E., Kakkar, V.V., Stalmans, I., Mattot, V., et al. 1999. Impaired myocardial angiogenesis and ischemic cardiomyopathy in mice lacking the vascular endothelial growth factor isoforms VEGF164 and VEGF188. Nat. Med. 5: 495-502.

Castellani, V. and Rougon, G. 2002. Control of semaphorin signaling. Curr. Opin. Neurobiol. 12: 532-541.

Cordes, S.P. 2001. Molecular genetics of cranial nerve development in mouse. Nat. Rev. Neurosci. 2: 611-623.

Davenne, M., Maconochie, M.K., Neun, R., Pattyn, A., Chambon, P., Krumlauf, R., and Rijli, F.M. 1999. Hoxa2 and Hoxb2 control dorsoventral patterns of neuronal development in the rostral hindbrain. Neuron 22: 677-691.

Dickson, B.J. 2002. Molecular mechanisms of axon guidance. Science 298: 1959-1964.

Ericson, J., Thor, S., Edlund, T., Jessell, T.M., and Yamada, T. 1992. Early stages of motor neuron differentiation revealed by expression of homeobox gene Islet-1. Science 256: 15551560.

Feiner, L., Webber, A.L., Brown, C.B., Lu, M.M., Jia, L., Feinstein, P., Mombaerts, P., Epstein, J.A., and Raper, J.A. 2001. Targeted disruption of semaphorin $3 \mathrm{C}$ leads to persistent truncus arteriosus and aortic arch interruption. Development 128: 3061-3070.

Feng, Y. and Walsh, C.A. 2001. Protein-protein interactions, cytoskeletal regulation and neuronal migration. Nat. Rev. Neurosci. 2: 408-416.

Gavalas, A., Ruhrberg, C., Livet, J., Henderson, C.E., and Krumlauf, R. 2003. Neuronal defects in the hindbrain of Hoxal, Hoxb1 and Hoxb2 mutants reflect regulatory interactions among these Hox genes. Development 130: 5663-5679.

Gerhardt, H., Golding, M., Fruttiger, M., Ruhrberg, C., Lundkvist, A., Abramsson, A., Jeltsch, M., Mitchell, C., Alitalo, K., Shima, D., et al. 2003. VEGF guides angiogenic sprouting utilizing endothelial tip cell filopodia. J. Cell Biol. 161: 1163-1177.

Gluzman-Poltorak, Z., Cohen, T., Herzog, Y., and Neufeld, G. 2000. Neuropilin-2 is a receptor for the vascular endothelial growth factor (VEGF) forms VEGF-145 and VEGF-165. I. Biol. Chem. 275: 18040-18045.

Gu, C., Limberg, B.J., Whitaker, G.B., Perman, B., Leahy, D.J., Rosenbaum, J.S., Ginty, D.D., and Kolodkin, A.L. 2002. Characterization of neuropilin-1 structural features that confer binding to semaphorin $3 \mathrm{~A}$ and vascular endothelial growth factor 165. J. Biol. Chem. 277: 18069-18076.

Gu, C., Rodriguez, E.R., Reimert, D.V., Shu, T., Fritzsch, B., Richards, L.J., Kolodkin, A.L., and Ginty, D.D. 2003. Neuropilin-1 conveys semaphorin and VEGF signaling during neural and cardiovascular development. Dev. Cell 5: 45-57.

Hafezparast, M., Klocke, R., Ruhrberg, C., Marquardt, A., Ahmad-Annuar, A., Bowen, S., Lalli, G., Witherden, A.S., Hummerich, H., Nicholson, S., et al. 2003. Mutations in dynein link motor neuron degeneration to defects in retrograde transport. Science 300: 808-812.

Haigh, J.J., Morelli, P.I., Gerhardt, H., Haigh, K., Tsien, J., Damert, A., Miquerol, L., Muhlner, U., Klein, R., Ferrara, N., et al. 2003. Cortical and retinal defects caused by dosagedependent reductions in VEGF-A paracrine signaling. Dev. Biol. 262: 225-241.

Harris, W.A., Holt, C.E., and Bonhoeffer, F. 1987. Retinal axons with and without their somata, growing to and arborizing in the tectum of Xenopus embryos: A time-lapse video study of single fibres in vivo. Development 101: 123-133.

He, Z. and Tessier-Lavigne, M. 1997. Neuropilin is a receptor for the axonal chemorepellent Semaphorin III. Cell 90: 739-751.

Kawakami, A., Kitsukawa, T., Takagi, S., and Fujisawa, H. 1996. Developmentally regulated expression of a cell surface protein, neuropilin, in the mouse nervous system. J. Neurobiol. 29: $1-17$.

Kawasaki, T., Kitsukawa, T., Bekku, Y., Matsuda, Y., Sanbo, M., Yagi, T., and Fujisawa, H. 1999. A requirement for neuropilin-1 in embryonic vessel formation. Development 126: 4895-4902.

Kitsukawa, T., Shimizu, M., Sanbo, M., Hirata, T., Taniguchi, M., Bekku, Y., Yagi, T., and Fujisawa, H. 1997. Neuropilinsemaphorin III/D-mediated chemorepulsive signals play a crucial role in peripheral nerve projection in mice. Neuron 19: $995-1005$

Kolodkin, A.L., Levengood, D.V., Rowe, E.G., Tai, Y.T., Giger, R.J., and Ginty, D.D. 1997. Neuropilin is a semaphorin III receptor. Cell 90: 753-762.

Lambrechts, D., Storkebaum, E., Morimoto, M., Del-Favero, J., Desmet, F., Marklund, S.L., Wyns, S., Thijs, V., Andersson, J., van Marion, I., et al. 2003. VEGF is a modifier of amyotrophic lateral sclerosis in mice and humans and protects motoneurons against ischemic death. Nat. Genet. 34: 383394.

Levy, A.P., Levy, N.S., Wegner, S., and Goldberg, M.A. 1995. Transcriptional regulation of the rat vascular endothelial growth factor gene by hypoxia. J. Biol. Chem. 270: 1333313340.

Maina, F., Hilton, M.C., Ponzetto, C., Davies, A.M., and Klein, R. 1997. Met receptor signaling is required for sensory nerve development and HGF promotes axonal growth and survival of sensory neurons. Genes \& Dev. 11: 3341-3350.

Mamluk, R., Gechtman, Z., Kutcher, M.E., Gasiunas, N., Gallagher, J., and Klagsbrun, M. 2002. Neuropilin-1 binds vascular endothelial growth factor 165, placenta growth factor2, and heparin via its b1b2 domain. J. Biol. Chem. 277: $24818-24825$.

Marin, O. and Rubenstein, J.L. 2003. Cell migration in the forebrain. Annu. Rev. Neurosci. 26: 441-483.

Marin, O., Yaron, A., Bagri, A., Tessier-Lavigne, M., and Rubenstein, J.L. 2001. Sorting of striatal and cortical interneurons regulated by semaphorin-neuropilin interactions. Science 293: $872-875$.

Miao, H.Q., Soker, S., Feiner, L., Alonso, J.L., Raper, J.A., and Klagsbrun, M. 1999. Neuropilin-1 mediates collapsin-1/ semaphorin III inhibition of endothelial cell motility: Functional competition of collapsin-1 and vascular endothelial growth factor-165. J. Cell Biol. 146: 233-242.

Miquerol, L., Gertsenstein, M., Harpal, K., Rossant, J., and Nagy, A. 1999. Multiple developmental roles of VEGF suggested by a LacZ-tagged allele. Dev. Biol. 212: 307-322.

Ohshima, T. and Mikoshiba, K. 2002. Reelin signaling and Cdk5 in the control of neuronal positioning. Mol. Neurobiol. 26: $153-166$.

Ohshima, T., Ogawa, M., Takeuchi, K., Takahashi, S., Kulkarni, A.B., and Mikoshiba, K. 2002. Cyclin-dependent kinase 5/p35 contributes synergistically with Reelin/Dab1 to the positioning of facial branchiomotor and inferior olive neurons in the developing mouse hindbrain. I. Neurosci. 22: 4036-4044.

Oosthuyse, B., Moons, L., Storkebaum, E., Beck, H., Nuyens, D., Brusselmans, K., Van Dorpe, J., Hellings, P., Gorselink, M., Heymans, S., et al. 2001. Deletion of the hypoxia-response element in the vascular endothelial growth factor promoter 
Schwarz et al.

causes motor neuron degeneration. Nat. Genet. 28: 131-138.

Pattyn, A., Hirsch, M., Goridis, C., and Brunet, J.F. 2000. Control of hindbrain motor neuron differentiation by the homeobox gene Phox2b. Development 127: 1349-1358.

Raper, J.A. 2000. Semaphorins and their receptors in vertebrates and invertebrates. Curr. Opin. Neurobiol. 10: 88-94.

Rice, D.S. and Curran, T. 2001. Role of the reelin signaling pathway in central nervous system development. Annu. Rev. Neurosci. 24: 1005-1039.

Riddle, R.D., Johnson, R.L., Laufer, E., and Tabin, C. 1993. Sonic hedgehog mediates the polarizing activity of the ZPA. Cell 75: 1401-1416.

Ruhrberg, C. 2003. Growing and shaping the vascular tree: Multiple roles for VEGF. Bioessays 25: 1052-1060.

Ruhrberg, C., Gerhardt, H., Golding, M., Watson, R., Ioannidou, S., Fujisawa, H., Betsholtz, C., and Shima, D.T. 2002. Spatially restricted patterning cues provided by heparin-binding VEGF-A control blood vessel branching morphogenesis. Genes \& Dev. 16: 2684-2698.

Sasaki, Y., Cheng, C., Uchida, Y., Nakajima, O., Ohshima, T., Yagi, T., Taniguchi, M., Nakayama, T., Kishida, R., Kudo, Y., et al. 2002. Fyn and Cdk5 mediate semaphorin-3A signaling, which is involved in regulation of dendrite orientation in cerebral cortex. Neuron 35: 907-920.

Soker, S., Takashima, S., Miao, H.Q., Neufeld, G., and Klagsbrun, M. 1998. Neuropilin-1 is expressed by endothelial and tumor cells as an isoform-specific receptor for vascular endothelial growth factor. Cell 92: 735-745.

Stalmans, I., Ng, Y.S., Rohan, R., Fruttiger, M., Bouche, A., Yuce, A., Fujisawa, H., Hermans, B., Shani, M., Jansen, S., et al. 2002. Arteriolar and venular patterning in retinas of mice selectively expressing VEGF isoforms. I. Clin. Invest. 109: 327-336.

Studer, M., Lumsden, A., Ariza-McNaughton, L., Bradley, A., and Krumlauf, R. 1996. Altered segmental identity and abnormal migration of motor neurons in mice lacking Hoxb-1. Nature 384: 630-634.

Takashima, S., Kitakaze, M., Asakura, M., Asanuma, H., Sanada, S., Tashiro, F., Niwa, H., Miyazaki Ji, J., Hirota, S., Kitamura, Y., et al. 2002. Targeting of both mouse neuropilin-1 and neuropilin-2 genes severely impairs developmental yolk sac and embryonic angiogenesis. Proc. Natl. Acad. Sci. 99: $3657-3662$.

Tamamaki, N., Fujimori, K., Nojyo, Y., Kaneko, T., and Takauji, R. 2003. Evidence that Sema3A and Sema3F regulate the migration of GABAergic neurons in the developing neocortex. J. Comp. Neurol. 455: 238-248.

Taniguchi, M., Yuasa, S., Fujisawa, H., Naruse, I., Saga, S., Mishina, M., and Yagi, T. 1997. Disruption of semaphorin III/D gene causes severe abnormality in peripheral nerve projection. Neuron 19: 519-530.

Tessier-Lavigne, M. and Goodman, C.S. 1996. The molecular biology of axon guidance. Science 274: 1123-1133.

Tiret, L., Le Mouellic, H., Maury, M., and Brulet, P. 1998. Increased apoptosis of motoneurons and altered somatotopic maps in the brachial spinal cord of Hoxc-8-deficient mice. Development 125: 279-291.

Varela-Echavarria, A., Tucker, A., Puschel, A.W., and Guthrie, S. 1997. Motor axon subpopulations respond differentially to the chemorepellents netrin-1 and semaphorin D. Neuron 18: 193-207.

Verzijl, H.T., van den Helm, B., Veldman, B., Hamel, B.C., Kuyt, L.P., Padberg, G.W., and Kremer, H. 1999. A second gene for autosomal dominant Mobius syndrome is localized to chromosome 10q, in a Dutch family. Am. I. Hum. Genet.
65: 752-756.

Yee, K.T., Simon, H.H., Tessier-Lavigne, M., and O'Leary, D.M. 1999. Extension of long leading processes and neuronal migration in the mammalian brain directed by the chemoattractant netrin-1. Neuron 24: 607-622.

Zhang, H., Vutskits, L., Pepper, M.S., and Kiss, J.Z. 2003. VEGF is a chemoattractant for FGF-2-stimulated neural progenitors. J. Cell Biol. 163: 1375-1384. 


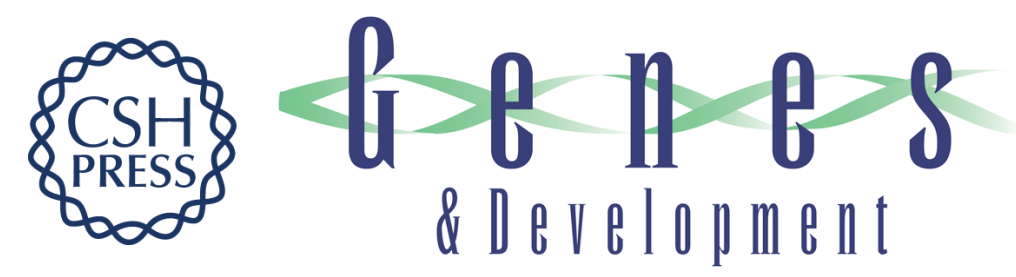

\section{Vascular endothelial growth factor controls neuronal migration and cooperates with Sema3A to pattern distinct compartments of the facial nerve}

Quenten Schwarz, Chenghua Gu, Hajime Fujisawa, et al.

Genes Dev. 2004, 18:

Access the most recent version at doi:10.1101/gad.322904

Supplemental

Material

References This article cites 57 articles, 24 of which can be accessed free at: http://genesdev.cshlp.org/content/18/22/2822.full.html\#ref-list-1

License

Email Alerting Service

http://genesdev.cshlp.org/content/suppl/2004/10/22/18.22.2822.DC1

Receive free email alerts when new articles cite this article - sign up in the box at the top right corner of the article or click here. 